\title{
Comprehensive Analysis on Anomalous Phenomenon of Ethanol-Soluble PVB Membrane for Ethanol Recovery via Pervaporation
}

\author{
Junjun $\mathrm{Li}^{1}$, Mi Huang ${ }^{1}$, Ping Wei ${ }^{1}$, Yaqin Zhang ${ }^{2}$, Xuean Zhao ${ }^{1}$, Chunbo Liu ${ }^{3}$, Zhijun \\ Zhou $^{1}$, and Lin Zhang ${ }^{1}$ \\ ${ }^{1}$ Zhejiang University \\ ${ }^{2}$ Affiliation not available \\ ${ }^{3}$ China Tobacco Yunnan Industrial Corporation
}

August 1, 2021

\begin{abstract}
Poly(vinyl butyral) is selected as a promising ethanol-permselective membrane based the solubility parameter theory, however it exhibits anomalous water perm-selectivity in practical pervaporation process. Comprehensive analysis based on experimental and theoretical methods were carried out to explore the inherent mechanism of the anomalous performance. Firstly, sum frequency generation vibrational spectra and contact angle were developed to quantify the surface reconstruction of membrane in air and ethanol, which indicated that hydrophilic hydroxyl tended to expose on membrane surface with ethanol thus improved the membrane affinity to water. Meanwhile, swelling behaviors proved more water would accumulate in the ethanol swollen membrane. Furthermore, theoretical analysis in terms of sorption and diffusion process, based on the UNIFAC-FV model and Fujita free volume theory, confirmed the mechanism of anomalous phenomenon of poly(vinyl butyral) membrane. The comprehensive investigation was expected to provide insights into the basic separation mechanism of pervaporation process.
\end{abstract}

\section{Hosted file}

main document.docx available at https://authorea.com/users/428623/articles/532439comprehensive-analysis-on-anomalous-phenomenon-of-ethanol-soluble-pvb-membrane-forethanol-recovery-via-pervaporation 